\title{
Myasthenia Class II
}

National Cancer Institute

\section{Source}

National Cancer Institute. Myasthenia Class II. NCI Thesaurus. Code C112015.

Mild weakness affecting non-ocular muscles. May also have ocular muscle weakness. 